\title{
Construction of Mechanized Claw as a Teaching Method in Engineering
}

\author{
Gabriel Souza da Silva ${ }^{1}$, André Felipe Souza da Cruz $^{1}$, Filipe Cavalcanti Fernandes ${ }^{1}$, Alberto Chéquer Novais \\ Neto $^{1}$ and Wellington da Silva Fonseca ${ }^{2}$ \\ 1. Faculdade de Engenharia Elétrica, Universidade Federal do Pará, Campus Tucuruí, Tucuruí 68464-000, Brazil \\ 2. Faculdade de Ciência e Tecnologia, Universidade Federal do Pará, Campus Ananindeua, Ananindeua 67020-000, Brazil
}

Received: December 10, 2014 / Accepted: December 29, 2014 / Published: February 25, 2015.

\begin{abstract}
The labor market requires, increasingly, an updated and more professional preparation, mainly due to the rapid pace of scientific technical advancement means in the industry. From this point, it is important to encourage the development of the basic techniques of control systems, electronics and communication in order to produce knowledge in the mechatronic field and so apply them to industry. Considering the relevance of this subject, the students of Federal University of Pará—Campus Tucuruí through the Research Group of Electrical and Mechanical Systems apply their knowledge in multidisciplinary engineering fields in order to generate knowledge from creative techniques using low cost materials, together with the encouragement of social and environmental responsibility which is very important nowadays. At the same time, targeting the needs of the labor market, this work aims to foster in students the skills and requirements through team projects and innovative methods. Therefore, this paper presents a mechanical manipulator, where their operating characteristics are based on industrial robots in the literature, powered by DC (direct current) motors and fully developed from reusable materials. By adding a developed control from the Arduino, prototyping platform with an electronic strategic offers through the internet the long-distance control convenience.
\end{abstract}

Key words: Arduino, mechanized claw, low cost, industry.

\section{Introduction}

Activities only based on "problem solving" can often result in difficulties in inserting newly graduated engineers in the labor market. This happens because that "troubleshooting" is connected only to classroom issues, i.e., solving the results which are usually numeric, which ends up not arousing the student the ability to diagnose problems, which in reality is what the labor market requires. You can work around this problem through activities project-based, collaborative and focused on problems that can be achieved through an favorable environment and tools that teachers can create with their students, since these are effective ways to boost student learning [1, 2].

In view of the difficulties in obtaining tools for

Corresponding author: Gabriel Souza da Silva, undergraduation, research field: automation. E-mail: gabriel18.tuc@gmail.com. robotics education that is accessible economically, The Research Group of Electrical and Mechanical Systems from the Federal University of Pará-Campus Tucuruí develops devices using low cost and/or reused materials in order to improve the learning of students from various engineering fields, seeking to offer, especially the possibility of awakening skills and abilities that are critical to their professional growth and better preparation for the labor market, making it possible to optimize students' learning through practical experiences that will help them in their professional future [3].

In this project, we developed the robotics study involving an electronic responsible for power control sent to actuators and data transmission involved in the prototype control. Therefore, they are not purely mechanical. They are made with a minimum of automation that is developed using pre-defined 
commands entered in the memory of an Arduino micro controller.

To improve students' learning, this paper proposes the construction of a mechanical claw moved by DC motors and controlled by an Arduino microcontroller based device, which will bring an interdisciplinary experience from various areas such as: mechanics, electronics, automation, signal processing and others [4]. The paper is organized as follows: Section 2 discusses the methodology; Section 3 introduces the results; finally, Section 4 is the conclusions.

\section{Methodology}

The mechanical claw is a project in the field of automation based on the design of an industrial manipulator robot. Four basic components define the formation of an industrial robot, which are: mechanical manipulator, actuators, sensors and control unit [5].

However, this project is not controlled automatically and does not have human independence. This happens because you want to control this robot over the internet and offer the operator the ability to manipulate the desired system to the long-distance convenience. Therefore, this project was built following these characteristics, limited only to the fact that this robot has no sensors for not being autonomous.

Thus, the construction of mechanized claw was divided into stages so that the student can obtain a better understanding of each addressed area. The steps are: the manipulator assembly, circuit assembly and the operation algorithm construction.

\subsection{Materials Used}

Reusable materials are a great alternative to alleviate the difficulties of production parts, due to their economic advantage thus reducing the cost of projects [6]. Thus, the following materials for the construction of mechanized claw were used:

- deodorant bottles (aluminum);

- DC motors from DVD Player;

- wire conductor jacketed;
- bare aluminum wire;

- flip flop rubber;

- Arduino control unit.

\subsection{Assembly}

The mechanical manipulator was built with aluminum sheets, worked and molded from deodorant bottles. This material is important, because besides matching the structural elements of the claw, which must be rigid, it also features easy handling as regards their malleability characteristics. To form the sheets, the bottles were cut in the top and bottom, where the side of the cylinder was used for the blade molding. These sheets are called links, and these are connected together via joints, which may vary in different sizes according to their position on the handle.

The joints have as raw material aluminum wires that are inserted into drilled holes in the links and these joints tips were molded to become fixed in their positions, pressing them so that they are expand. This method has been used in various parts of the structure, but only two together offer degree of freedom for the mechanized claw, allowing two kinds of motion, up and down and allow the effect or to open and close.

To assemble the transmission system constituting the project, we used a system based on a mechanical jack. In a tube, with teeth inside, a worm thread screw is embedded. So, depending on the direction of its rotation, the screw is thrown out or taken into the tube. However, for the screw to rotate, an actuator that is used in DC motors coupled by a rubber pulley at the end of the transmission system is required.

The control unit chosen to be used in this project was the Arduino, a prototyping platform consisting basically of a microcontroller, an integrated programming language with a built development environment in Java and support for input and output data. The Arduino was chosen because it has very interesting advantages that have been adapted perfectly to this project, as the low cost compared to other control units and the simplicity when it comes to a 
quick learning $[7,8]$.

\subsection{Implemented Circuit}

The circuit consists of two "H-bridges" and an Ethernet shield, which are powered by a DC power source. The H-bridge is a simple circuit capable of controlling DC motors directly from electrical signals, allowing the rotation control to the power and speed control. These electrical signals are output by the Arduino in different PWM (pulse-width modulation) ranges depending on the information that is received over the internet through Ethernet shield.

Fig. 1 presents the elaborated electrical circuit, which represents the mechanized claw control circuit. In this circuit, we used eight NPN (negative-positive-negative) transistors together with eight resistors, responsible for allowing or not the current flow in the circuit, and the Ethernet shield, cited above, that enables the reading of these data by the user in an understandable way. The devices used were:

- eight TIP120 NPN transistors;

- Ethernet shield;

- eight $10 \mathrm{~K}$ ohms resistors.
The TIP 120 NPN transistors and the 10K resistors were connected to digital ports with Arduino PWM special functions because therethrough we could send information signals simulating discrete analog signals, that is, one can control the amount of power delivered by the engines transistors .

The Ethernet shield is responsible for connecting the Arduino to the internet simply using codes that are already inserted in the Arduino IDE (integrated development environment) itself and that make control of the shield, providing an IP (internet protocol) address to the Arduino, thus allowing it to work with TCP (transmission control protocol) and UDP (user datagram protocol). It also has standard network connection RJ-45, allowing the Arduino network connection [8].

\subsection{Implemented Algorithm}

First, a page in HTML (hypertext markup language) was created that is hosted on a server, in this case the Ethernet shield attached to the Arduino. The elaborate logic was accomplished through the Arduino IDE, that when it receives information from the hosted

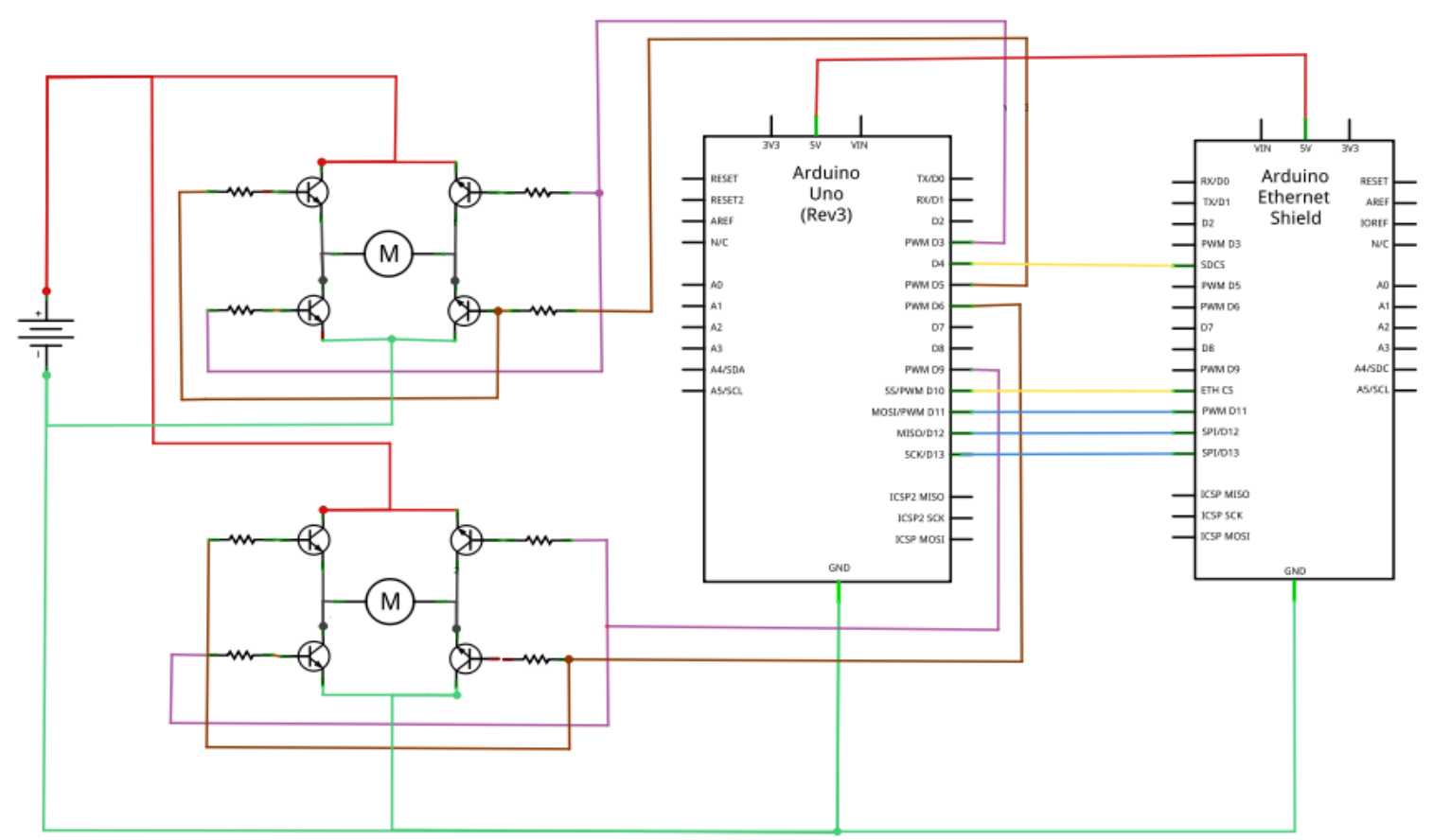

Fig. 1 Circuit implemented to control the mechanized claw. 


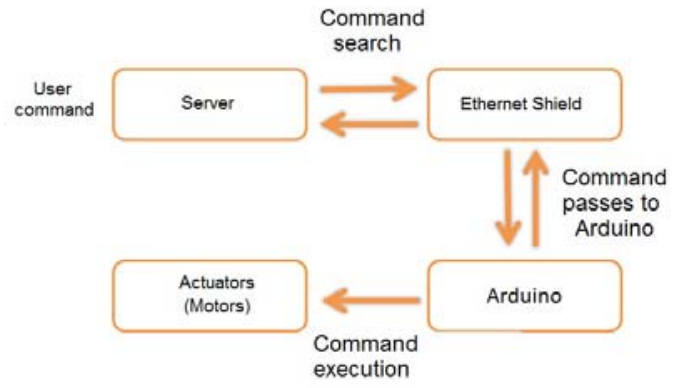

Fig. 2 Algorithm implemented to control the mechanized claw.

page takes determine predefined actions according to what was previously stored in its memory. Buttons were created on the page, each button sends information to the Arduino, which processes it and sends a response back to the page and to the actuators. The detailed scheme of the exchanges can be seen in Fig. 2. This logic happens through the following steps:

- The microcontroller starts the system, the required libraries, the variables and the defined constants;

- The Arduino processes the information that the user entered in the server, which sends the information to the Ethernet shield;

- Ethernet shield passes the information to the Arduino;

- The Arduino processes the information and takes the necessary decisions, signaling to the actuators.

\section{Results}

The use of team projects for engineering education has created a new possibility of learning, since this methodology allowed direct contact of students with new concepts that will help them in their understanding of engineering techniques.

When working with the assembly of the claw, the students learned to design because it was necessary to study how the claw structure would be and which materials would be used in its construction, and from these studies they had to run it, thus arousing skills related to research and knowledge in various mechanics fields.

Having in hand the mechanical design, the new

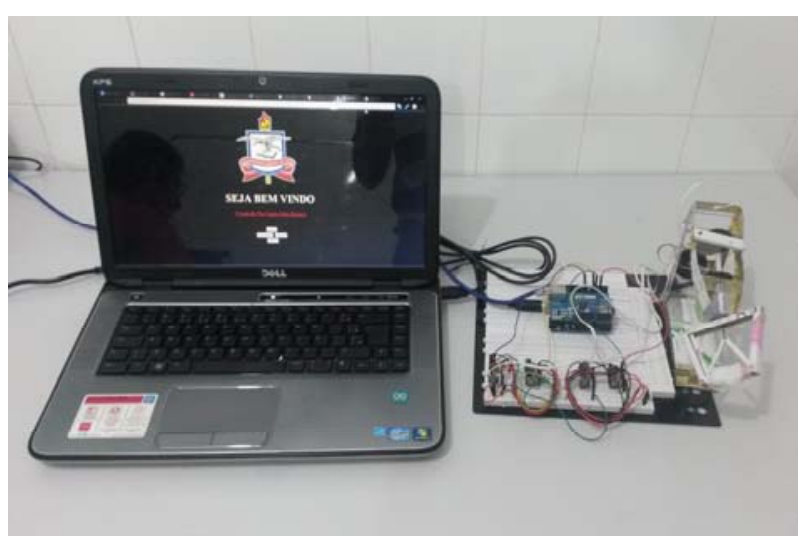

Fig. 3 To the left, it is the web page, to the right it is the mechanized claw and the electrical circuit.

challenge for the students was to design an electronic circuit and a computer program that was able to control it.

Since the mechanized claw actuators were motors, the most efficient way to control this type of device was researched. There was a great learning experience in relation to overcoming problems because some devices were not easily access, so the learners could manage to adapt the original design with the new difficulty. Deepening their skills to overcome problems is besides absorbing knowledge on electrical and electronics fields.

When creating the program for the microcontroller and the web page that would send commands to the circuit, it was an enriching step due to the proposal that came from the students themselves to establish a communication from a long distance with the device, thereby encouraging proactivity, being a spontaneous learning.

Fig. 3 shows the completed mechanized claw design, where you can see each of the connected steps forming a single set.

\section{Conclusions}

The work had as main goal offering a new method of teaching based on projects because it was realized that the teaching of classical methods ends up discouraging many students of the course of engineering. Thus, this new method was very effective, 
since it was observed that the students were able to learn much of the content in the areas that the project addressed mainly to the points that generated more difficulties, because the students had to search to overcome these situations and find the solutions necessary for the operation of the project.

\section{References}

[1] Belhot, R. V. 1997. "Reflection and Purpose about “Teaching Engineering” in the 21th Century.” Ph.D. thesis, University of São Paulo.

[2] Reis, L. R. 2011. "Active Learning Strategies to Reduce School Failure: Role of the Educational Psychologist.” Teaching Portfolio for Higher Education. Accessed May 28, 2014. http://portfoliodedidaticadoensino2011.blogspot. com.br.

[3] Lima, M. E. C. C., Júnior, O. G. A., and Braga, S. A. M. 1999. Learning Science: A World of Materials. Belo Horizonte: UFMG (Federal University of Minas Gerais).

[4] Carrara, V. 1999. "Robotic Handout.” University of Braz Cubas. Accessed May 26, 2014. http://www.valcar. net/cursos/rb_apostila.pdf.

[5] Romano, V. F. 2002. Introduction to Industrial Robotics. Rio de Janeiro: Editora Blucher.

[6] Ferreira, G. A., and Miguelote, A. Y. 2010. "Learning Physical Concepts by Building Experiments with Low Cost Materials.” University Unigranrio.

[7] Fonseca, E. G. P., and Veja, A. S. 2011. "Introduction Tutorial about the Project Using the Arduino Development Kit.” Presented at the XXXIX Brazilian Congress on Engineering Education, FURB, Blumenau, Braizl.

[8] Arduino. Accessed May 2014. http://arduino.cc/. 